\title{
International Year of Chemistry 2011
}

SCS

Division of

Industrial Chemistry

\section{Popular Scientific Papers}

Presented by the Division of Industrial Chemistry

\section{EMS - Der führende Spezialist für Hochleistungspolyamide}

\section{Stephan Wick and Christian Kruse*}

${ }^{\star}$ Correspondence: C. Kruse, Manager Application Development Centre, EMS-CHEMIE AG, Business Unit EMS-GRIVORY, Via Innovativa 1, CH-7013 Domat/Ems, Tel.: +41 81632 71 61, Fax: + 418163274 10, E-mail: Christian.Kruse@emsgrivory.com

Abstract: This short review introduces EMS-Chemie as a leading producer of high-performance polyamides. The history of polyamides is described as well as their innovative development and modification for customized applications.

Keywords: EMS-Chemie · High-performance polyamides . Metal substitutes $\cdot$ Reinforcing materials

\section{75 Jahre EMS-CHEMIE}

Die EMS-CHEMIE (Bild 1) feiert dieses Jahr ihr 75-jähriges Jubiläum. 1936 gründete Dr. Werner Oswald die HOLZVERZUCKERUNGS AG (HOVAG) mit Sitz in Zürich (Schweiz), um mit dem 'Schollerschen Verfahren' aus Holz Aethylalkohol als Ersatz-Treibstoff für Motorfahrzeuge und Flugzeuge herzustellen. Die Produktion des sogenannten 'Emser Wassers' wurde im Herbst 1942 in Domat/Ems (Kanton Graubünden) mit einheimischen Holzabfällen in Betrieb genommen. 'Emser Wasser' deckte bis Kriegsende rund 30\% des schweizerischen Treibstoffbedarfs und wurde bis 1956 produziert. Durch den Entscheid des Schweizer Volkes am 13. Mai 1956, die subventionierte Abnahme von 'Emser Wasser' als Treibstoffersatz durch den Bund nicht weiterzuführen, musste die Produktion eingestellt werden. In der Folge wurde die HOVAG in EMSER WERKE AG umbenannt (Bild 2).

Neu konzentrierte sich das Unternehmen auf die Produktion und Vermarktung von synthetischen Textilfasern aus Grilon ${ }^{\circledR}$ (Polyamid 6 / Nylon), welches aus seit 1951 eigenproduziertem Caprolactam hergestellt wurde. 'Grilon' ist eine Wortkombination aus 'Grison' (französisch für 'Graubünden') und 'Nylon'. Grilon ${ }^{\circledR}$-Textil-Fasern waren bekannt für ihre besondere Robustheit und veränderten damals die Textilwelt. In den 80er-Jahren

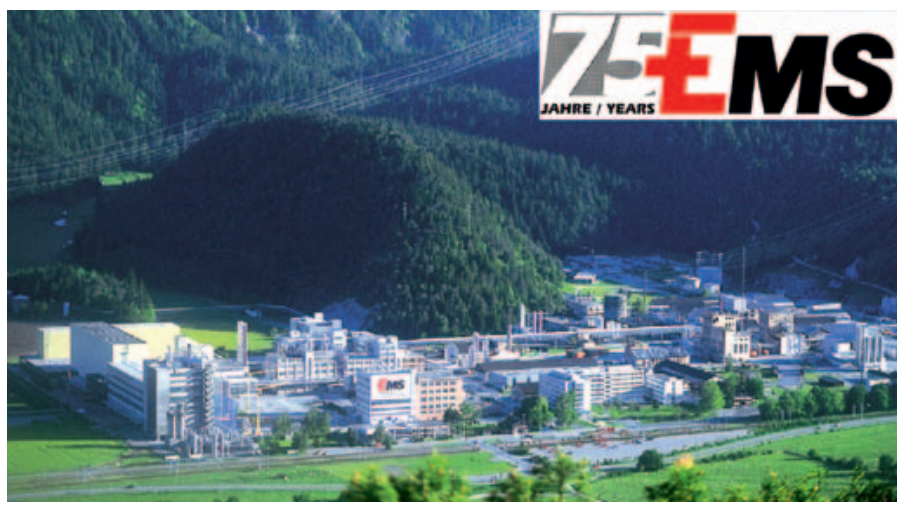

Bild 1. EMS-CHEMIE, in Domat/Ems (Schweiz).

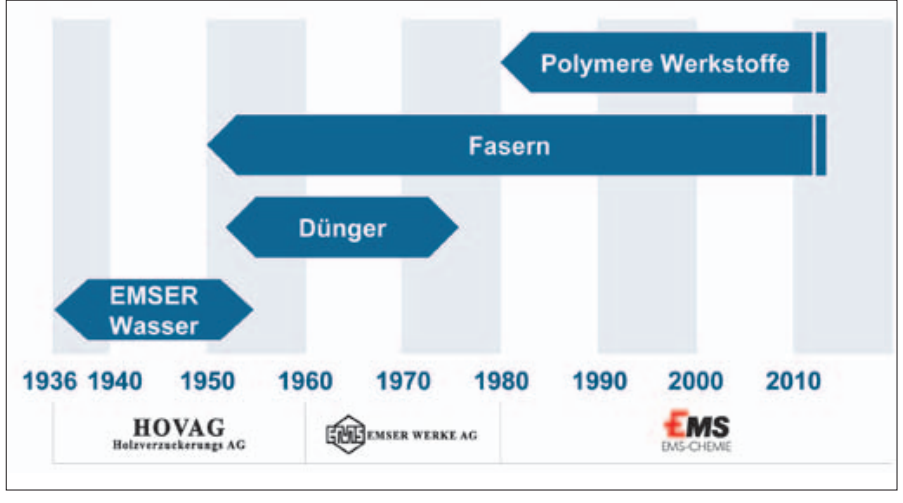

Bild 2. Die Geschichte der EMS-CHEMIE AG.

stellte EMS die Produktion von Textilfasern ein, nachdem sich die Textilindustrie zunehmend nach Asien verlagerte. Als technische Spezialfasern, z.B. in der Herstellung von Papiermaschinenfilzen, gehören Grilon ${ }^{\circledR}$-Produkte auch heute noch zum Produktsortiment von EMS-CHEMIE.

Mit der Produktion von Caprolactam startete auch die Düngemittelproduktion. Bei der Produktion von Caprolactam fällt Ammonsulfat als Nebenprodukt an, welches sich als Stickstoffquelle gut als Dünger für die Landwirtschaft eignete. Als weitaus besserer Dünger wurde auch Harnstoff in einem von EMS entwickelten Verfahren hergestellt. Die Produktion von Caprolactam wurde bis 1974 und der Verkauf von Dünger bis 1988 aufrechterhalten. In den Folgejahren bis 1980 wurden viele weitere Produkte entwickelt: Grilonit ${ }^{\circledR}$-Epoxidharze für die Bau- und Lackindustrie (1963); Polyesterfasern Grilene ${ }^{\circledR}$ (1964), Grilamid ${ }^{\circledR}$-Polyamid 12 und Grilon ${ }^{\circledR}$-Co-Polyamid für die Herstellung von Kunststoffrohren und Verpackungsfolien (1966), Griltex ${ }^{\circledR}$-Co-Polyamid SchmelzklebepulverfürdieBeschichtung vontextilenEinlagenfür die Konfektionsindustrie (1967) sowie Grilesta ${ }^{\circledR}$-Polyester-Harze für die Herstellung von Pulverlacken zur Metallbeschichtung (1980).

1981 erfolgte die Namensänderung von EMSER WERKE AG zu EMS-CHEMIE AG und damit auch eine Konzentration auf die Entwicklung und Vermarktung von Polymeren Werkstoffen.

1991 lancierte EMS bei den Polymeren Werkstoffen mit Grivory ${ }^{\circledR} \mathrm{GV}$ eine neue Generation von hochsteifen und hochfesten Polyamiden und läutete damit die Ära des Metallersatzes ein. Diese wurde im Jahr 1995 erweitert mit der HochtemperaturPolyamidfamilie Grivory ${ }^{\circledR}$ HT für den Einsatz bei hohen Gebrauchstemperaturen. EMS nahm damit die erste und bis heute einzige Produktionsanlage für Hochtemperatur-Polyamide in Europa in Betrieb. 2008 stieg EMS in die Produktion von Langglasfaser verstärkten Polyamiden (LFT) ein und bietet Grivory ${ }^{\circledR}$ und Grilon ${ }^{\circledR}$-Typen mit Langglasfasern an. Die Kombination von Steifigkeit und Schlagzähigkeit erweitert das Einsatzspektrum beträchtlich und ermöglicht den Einsatz in Anwendungen, wo Crashresistenz gefordert wird. Grivory ${ }^{\circledR}$ HT3 ist die aktuellste Entwicklung im Bereich der Hochtemperaturpolyamide auf Basis von nachwachsenden Rohstoffen für die Erschliessung von neuen Märkten mit der Forderung nach Bio-Polymeren. 


\section{Der Werkstoff Polyamid}

Polyamide (Kurzzeichen PA) sind Polymere, deren Wiederholungseinheiten als charakteristisches Merkmal die Amidgruppe besitzen. Die Amidgruppe kann als Kondensationsprodukt einer Carbonsäure und eines Amins aufgefasst werden. Die dabei entstehende Bindung nennt man Amidbindung. Sie ist hydrolytisch wieder spaltbar.

Die Bezeichnung Polyamide wird üblicherweise als Bezeichnung für synthetische, technisch verwendbare thermoplastische Kunststoffe verwendet und grenzt diese Stoffklasse damit von den chemisch verwandten Proteinen ab. Fast alle bedeutsamen Polyamide leiten sich von primären Aminen ab, das heisst in ihren Wiederholeinheiten kommt die funktionelle Gruppe -CONH- vor. Daneben existieren auch Polyamide von sekundären Aminen (-CO-NR-, R = organischer Rest). Als Monomere für die Polyamide finden besonders Aminocarbonsäuren, Lactame und/oder Diamine und Dicarbonsäuren Verwendung. Die Monomere bestimmen u.a. die chemische Beständigkeit, die Zähigkeit, die Wärmeform- und Dimensionsbeständigkeit und das Barriereverhalten.

Erste systematische Untersuchungen zu den Produkten aus der Polykondensation von Dicarbonsäuren mit Diaminen wurden in den 30er Jahren von Wallace H. Carothers bei DuPont durchgeführt. Eine erste Produktionsanlage für Polyamid 66 (Nylon) aus Hexamethylendiamin und Adipinsäure wurde 1939 in Betrieb genommen (Bild 3).

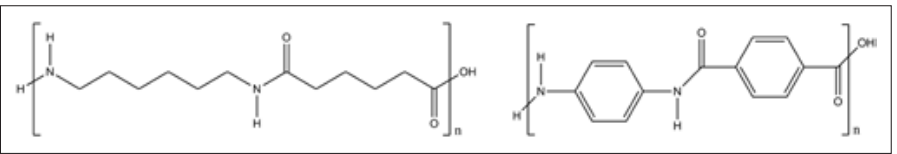

Bild 3. Strukturformel für aliphatische und rein aromatische Polyamide.

Die hydrolytische Polymerisation des e-Caprolactams gelang Paul Schlack in Stuttgart erstmals im Jahr 1937. Die Entwicklung weiterer Polyamide zur Produktionsreife setzte im Anschluss an die Arbeiten von Carothers insbesondere nach Ende des Zweiten Weltkriegs ein. Dazu gehören Polyamide und Copolyamide mit hohen Schmelztemperaturen, transparente oder lösliche Polyamide, Copolyamide mit niedrigem Schmelzpunkt für die Anwendung als Schmelzkleber und elastomere Blockcopolyamide. Die rein aromatischen Polyamide (Aramide) sind thermoplastisch nicht mehr verarbeitbar und zeichnen sich durch eine sehr hohe thermische und chemische Beständigkeit und gute Festigkeiten aus.

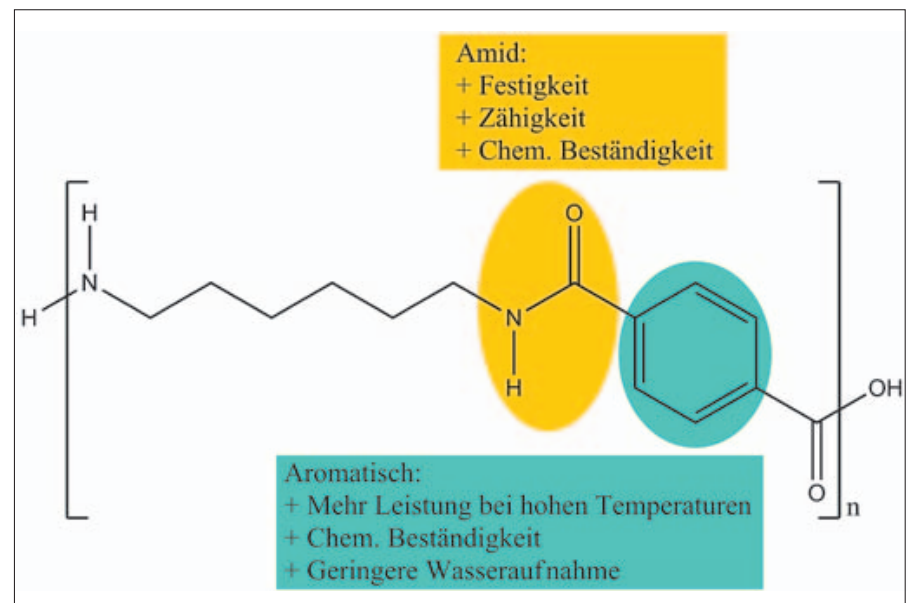

Bild 4. Strukturformel und Eigenschaftsprofil für Grivory HT.
Anfang der 90er Jahre, wurden unter anderem auch von EMS teilaromatische Polyamide (PPA) als neue Generation von Hochleistungs- und Hochtemperaturwerkstoffen auf den Markt gebracht. Teilaromatische Polyamide widerstehen Hitze, Chemikalien und Feuchtigkeit deutlich besser als die klassischen PA 66-Typen. Bestimmend für diese spezifischen Eigenschaften sind die bei der Polymerisation verwendeten aromatischen Monomere wie Isophthal- oder Terephthalsäure sowie die Wahl des aliphatischen Bestandteils bei der Copolymerisation, z. B. PA 66. Je grösser bei solchen Werkstoffen der aromatische Anteil, z. B. an PA 6T, im Verhältnis zum aliphatischen PA 66 ist, umso höher ist die resultierende Glasübergangstemperatur (Bild 4).

\section{Mit Verstärkungsstoffen zu metallähnlichen Eigenschaften}

Füll- und Verstärkungsstoffe sind feste Zusatzstoffe, die in Kunststoffe eingearbeitet werden, um die Materialeigenschaften im gewünschten Sinne zu verbessern. Es handelt sich dabei um teilchenförmige Partikel, die organischer (polymerer) oder anorganischer Natur sein können und die in der sie umgebenden Polymermatrix dispergiert sind. Diese Füllstoffe führen zu einer Veränderung der Materialeigenschaften der Polymermatrix. Beim Einsatz von Füll- und Verstärkungsstoffen werden Dichte, Härte, Steifigkeit sowie Wärmeformbeständigkeit erhöht. Die Begriffe 'Füllstoffe' und 'Verstärkungsstoffe' sind dabei häufig nicht klar voneinander abgegrenzt. In der Regel unterscheidet man zwischen inerten Füllstoffen, die lediglich zum Strecken der Matrixpolymeren und damit zur Verbilligung des Produkts eingesetzt werden und den verstärkenden Füllstoffen (funktionelle Füll- bzw. Verstärkungsstoffe), die zusätzlich zu den bereits erwähnten Eigenschaftsveränderungen auch die Festigkeit der Mischung im Vergleich zu der des Matrixpolymers anheben. Die Eigenschaften verstärkter Thermoplaste werden hauptsächlich vom Volumenanteil des Verstärkungsstoffes, dessen Form (Länge/Durchmesser-, L/D- oder Aspektverhältnis) und der Wechselwirkung an der Grenze zur Polymermatrix beeinflusst.

Der für die mechanischen Eigenschaften des Verbunds bedeutsame Formfaktor ist definiert als das Verhältnis seiner Länge zu seiner Dicke (L/D-Verhältnis). Kugeln und kubische Partikel (Calciumcarbonat, Glaskugeln) haben einen Formfaktor von 1, während Fasern oder andere anisotrope plättchenförmige Füllstoffe (Schichtsilikate wie Talk und Glimmer) mit einem grossen L/D-Verhältnis sehr hohe Formfaktoren aufweisen und damit die Polymermatrix deutlich versteifen (Bild 5).

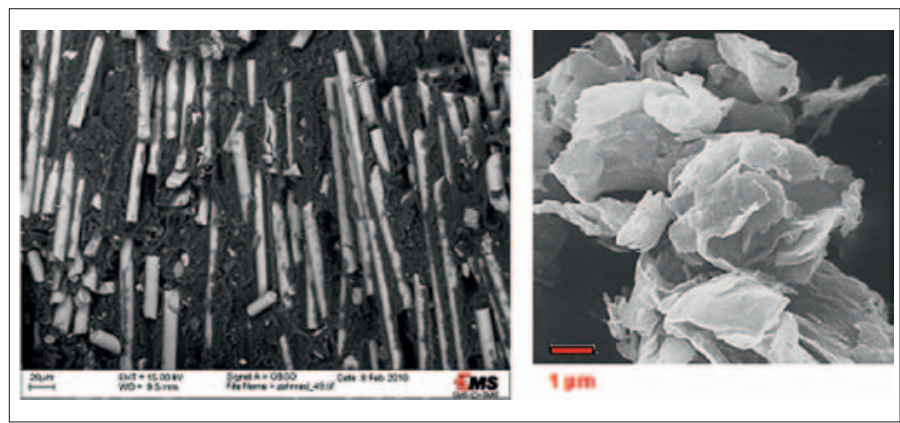

Bild 5. Verstärkungsstoffe Glasfasern und Schichtsilikate.

Eine angelegte mechanische Spannung wird von der Polymermatrix aufgenommen und auf den Füllstoff übertragen. Je grösser das Aspektverhältnis des Füllstoffes ist, desto besser kann die durch die Spannung verursachte Energie im Material abgeführt werden. Eine Beschichtung der Füllstoffpartikel mit organi- 
schen Kupplungsreagenzien verbessert die Verträglichkeit mit der Matrix, die Verarbeitbarkeit und auch die resultierenden mechanischen Festigkeiten. So kann z.B. durch die Reaktion von aminosilanbeschichteten Füllstoffpartikeln mit Polyamiden die Phasenanbindung deutlich verbessert werden.

\section{Hochleistungspolyamide von EMS substituieren Metall}

Wie in der Geschichte von EMS aufgezeigt, startete EMS bereits 1991 mit Grivory ${ }^{\circledR}$ GV erfolgreich die Ära des Metallersatzes (Bild 6). In den letzten Jahren wurden die Produkte in ihrer Leistungsfähigkeit für deutlich höhere Gebrauchstemperaturen und/ oder metallähnlichere Festigkeiten gezielt weiterentwickelt.

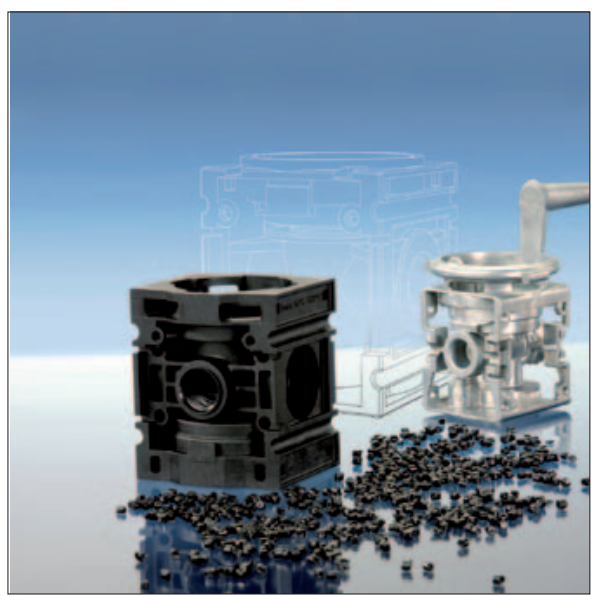

Bild 6. Polyamide ersetzen immer öfter herkömmliche Druckgussmetalle.

Grivory HT, ein teilkristalliner thermoplastischer Konstruktionswerkstoff auf Basis von Polyphthalamid PPA, ist die Produktfamilie für deutlich höhere Gebrauchstemperaturen. Für die Polymerisation und die Compoundierung von Grivory HT entwickelte EMS in Domat/Ems ein eigenes, neues Verfahren.

Neue, speziell faserverstärkte Polyamide eröffnen zusätzliche ungeahnte Möglichkeiten in der Metallsubstitution und stossen im Markt auf grosses Interesse. Als Beispiel dargestellt sind hier die im EMS-eigenen Pultrusionsverfahren hergestellten Langglasfaserprodukte Grivory GVL und Grilon TSGL. Durch die Verwendung von Langglasfasern eignen sich diese Produkte noch besser für den Metallersatz. Sie sind sowohl von der Wirtschaftlichkeit als auch von der Performance her echte Alternativen zu Druckgussmetallen. Mit ihren erstaunlich hohen Festigkeitswerten sind sie an der Spitze der thermoplastischen Kunststoffe (der technischen Kunststoffe) anzusiedeln. Durch die bis zu $10 \mathrm{~mm}$ langen Glasfasern im Polyamid entsteht im Bauteil ein Faserskelett,

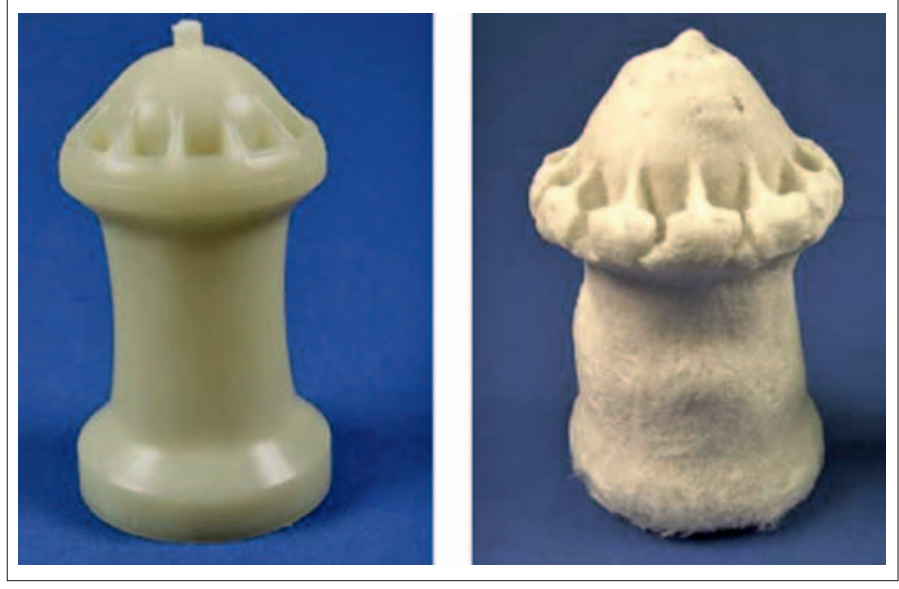

Bild 7. Verbleibende Langglasfaserstruktur nach dem Ausschmelzen der Polymermatrix.

welches hochbelastbare und hitzestabile Strukturbauteile ermöglicht. Durch diese filzartige Faserstruktur im Kern des Bauteils erhöht sich Kerbschlagzähigkeit, Wärmeformbeständigkeit und Energieaufnahme. Die Festigkeit bleibt auch bei steigenden Temperaturen auf einem deutlich höheren Niveau. Das Kriechen, das heisst die Verformung der Bauteile unter einer lang anhaltenden Belastung, ist auf ein absolutes Minimum reduziert (Bild 7).

Das heute erreichbare Leistungspotential dieser Werkstoffe beweist ein unscheinbarer Gewindetubus aus langglasfaserverstärktem Polyamid, der locker einen PKW in die Höhe hebt.

Das Gesamtgewicht des Fahrzeuges mit Aufhängevorrichtung betrug 1.8 Tonnen. Das ganze Gewicht wurde einerseits am Gewindetubus aus Polyamid und andererseits an einer $8 \mathrm{~mm}$ Schraube aus Stahl befestigt. Die ganze Belastung wird auf einen Querschnitt von nur rund 2,4 $\mathrm{cm}^{2}$ verteilt und die Anforderungen an den Tubus sind enorm hoch. Die Oberflächenhärte des Tubus muss dem scharfkantigen Gewinde der Schraube trotzen. Zudem kommt eine notwendige hohe Kriechbeständigkeit, damit sich das Kunststoffteil durch die Belastung nicht innerhalb weniger Sekunden so verformt, dass die Schraube ausreisst oder der Tubus bricht. Belastungstest in den Prüflabors nach DIN267T3 ergaben eine Maximalbelastung von knapp 3 Tonnen (Bild 8).

\section{Erfolgsrezept von EMS beim Metallersatz}

Doch das Erfolgsrezept von EMS beim Metallersatz basiert auf mehr als nur den geeigneten Werkstoffen. Neben einem breiten Angebot an Polyamiden, welche bis an die Grenzen der Metalleigenschaften herankommen, bietet EMS auch anwendungstechnische Dienstleistungen an. Dies beginnt meist schon bei der Bauteilgestaltung. EMS-GRIVORY verfügt über eine eigene
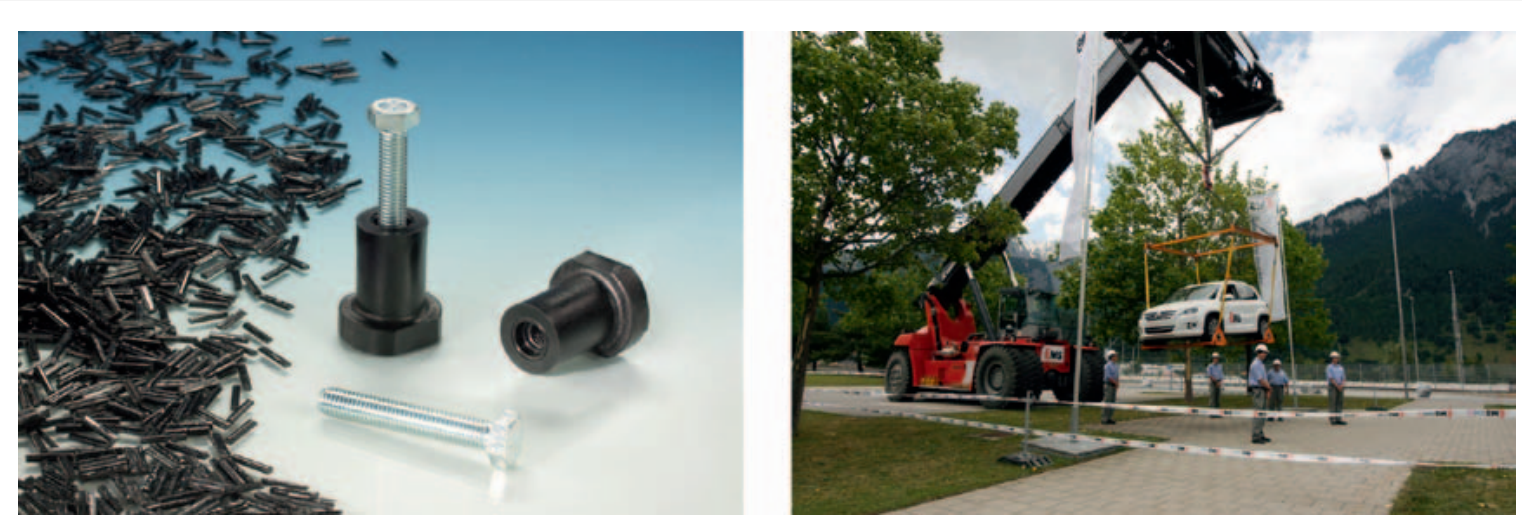

Bild 8. PKW wird an einem $8 \mathrm{~mm}$ Gewindetubus aus Polyamid aufgehängt. 


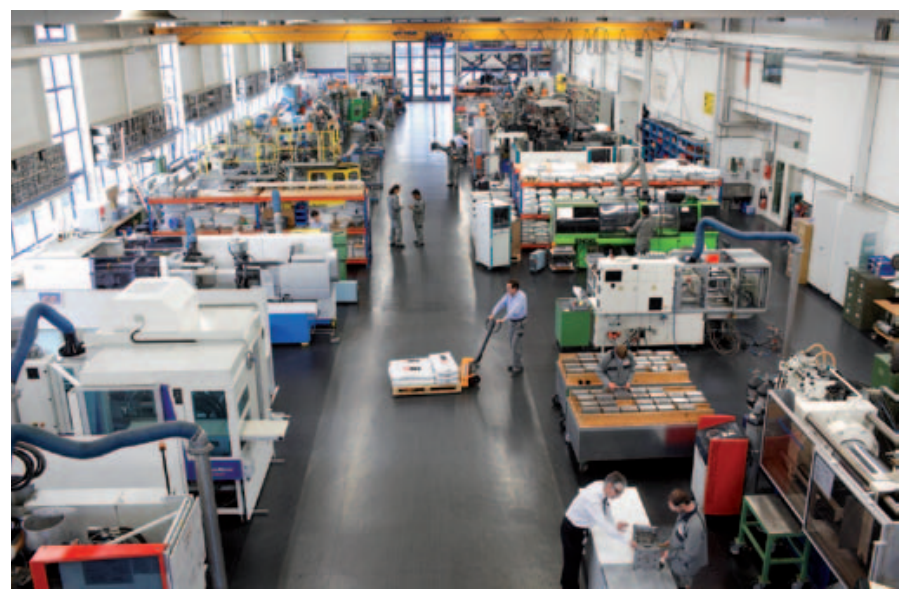

Bild 9. Blick in die Anwendungstechnik von EMS-GRIVORY.

CAE-Abteilung (Computer Aided Engineering), welche vorab Machbarkeitsstudien und Designvorschläge ausarbeitet. Nur selten können Druckgussbauteile ohne Modifikationen direkt in Kunststoff umgesetzt werden. Hier helfen Finite-Elemente-Berechnungen oder rheologische Simulationen bereits im Vorfeld der Werkzeugherstellung. Die aus den Simulationen gewonnenen Erkenntnisse fliessen in den Werkzeugbau ein und helfen so teure Fehler und Werkzeugkorrekturen zu vermeiden.

Neben den Berechnungsdienstleistungen bietet EMS mit der Anwendungstechnik weltweit eine aktive und umfassende Unterstützung bei der Entwicklung von neuen Anwendungen aus Polyamiden. Erfahrene Anwendungsentwickler helfen bei der richtigen Materialwahl, erstellen Kostenvergleiche (z.B. beim
Ersatz von Druckgussmetallen) und stehen der kunststoffverarbeitenden Industrie mit Expertenwissen zur Seite. Der umfangreiche Maschinenpark der Anwendungstechnik ermöglicht interne Materialtests sowie die Produktion von Prototypen für Kunden. So bietet EMS unter anderem den Service, Polyamide auf bestehenden Druckgusswerkzeugen der Kunden zu verarbeiten. Dabei können bereits einfache Änderungen des Designs oder auch kunststoffgerechte Anpassungen beim Angusssystem berücksichtigt werden (Bild 9).

Die optimale Produktpalette in Kombination mit einer leistungsfähigen Anwendungstechnik bietet dem Kunden ein Leistungspaket, das von der ersten Idee bis zur Serienproduktion reicht.

\section{Ausblick}

Die Produkte von EMS basieren auf einer sehr breiten Palette von Basis-Polymeren, die heute kein anderer Polyamid-Produzent anbieten kann. Die hochverstärkten Werkstoffe Grivory haben sich seit vielen Jahren ausgezeichnet beim Ersatz von Druckgussmetallen bewährt. Die Integration von Funktionen wie Lager oder Verbindungselemente sowie der Verzicht auf Nachbehandlungen wie spanendes Bearbeiten, Lackieren oder Korrosionsschutz spart aufwändige Fertigungsschritte und reduziert die Fertigungskosten. Durch die bis zu viermal geringere Dichte der EMS-Kunststoffe sinken die Transportkosten der Teile und bei Anwendungen im Automobilbereich der Treibstoffverbrauch und somit der $\mathrm{CO}_{2}$-Ausstoss.

Eingegangen am 4. April 2011 